\title{
Intermixing and shape changes during the formation of InAs self-assembled quantum dots
}

\author{
J. M. García, a) G. Medeiros-Ribeiro, ${ }^{\text {b) }}$ K. Schmidt, ${ }^{\text {() }}$ T. Ngo, J. L. Feng, ${ }^{\text {a) }}$ A. Lorke, ${ }^{\text {d) }}$ \\ J. Kotthaus, ${ }^{d)}$ and P. M. Petroff \\ Materials Department and QUEST, University of California, Santa Barbara, California 93106
}

(Received 19 March 1997; accepted for publication 7 August 1997)

\begin{abstract}
The initial stages of GaAs overgrowth over self-assembled coherently strained InAs quantum dots (QDs) are studied. For small GaAs coverages (below $5 \mathrm{~nm}$ ), atomic force microscopy (AFM) images show partially covered island structures with a regular size distribution which are elongated in the [011] direction. Analysis of the AFM profiles show that a large anisotropic redistribution of the island material is taking place during the initial GaAs overgrowth. Short time annealing experiments together with photoluminescence spectroscopy on annealed QDs are consistent with a $\mathrm{Ga}$ and In intermixing during the overgrowth. Surface QDs capped with $5 \mathrm{~nm}$ or more GaAs show a strong luminescence intensity indicating that surface QDs are remarkably insensitive to surface recombination effects. (C) 1997 American Institute of Physics. [S0003-6951(97)01040-1]
\end{abstract}

The electronic and structural properties of selfassembled quantum dots (QDs) made by molecular beam epitaxy $(\mathrm{MBE})^{1,2}$ have been intensively investigated in the last few years. ${ }^{3}$ Size quantization effects have also been demonstrated. ${ }^{4}$ The electrons' energy levels have been measured on a single QD using ballistic electron microscopy (BEEM). ${ }^{5}$ Electron trapping in QDs ${ }^{6}$ and laser diodes based on a QD active layer have also been demonstrated. ${ }^{7,8}$

One of the unsolved problem in most of these studies has been comparison of the observed quantization effects with theory. ${ }^{9,10}$ Nonuniform size distribution of truncated pyramidal shape and uncertainties on the values of QDs height $(2-10 \mathrm{~nm})$ are partially responsible for this situation.

The usual size measurement ${ }^{11,12}$ technique uses atomic force microscopy (AFM) on uncapped islands of InAs. The growth kinetics during the overgrowth of the island by the capping material are complex and probably involve a materials redistribution between the wetting layer and the QDs. ${ }^{13,14}$

Previous studies of the QDs overgrowth process ${ }^{15}$ using $\left(\mathrm{Al}_{25} \mathrm{Ga}_{.75} \mathrm{As} / \mathrm{GaAs}\right)$ marker layers showed the existence of an important material redistribution during the capping operation. Some of the observed effects could have been introduced by the marker layer themselves since the group III elements (e.g., In or Ga) mobility is slowed down on an AlGaAs surface.

This letter addresses the kinetic processes that are operating while the coherently strained InAs islands are capped with the GaAs film and in the process are transformed into QDs.

The InAs islands are grown by MBE on Si doped GaAs (100) substrate without rotation ${ }^{4}$ and annealed for $40 \mathrm{~s}$ under $1 \times 10^{-5}$ Torr arsenic pressure at $530^{\circ} \mathrm{C}$ to narrow the island size distribution. ${ }^{16}$ The islands are capped with GaAs (from

\footnotetext{
${ }^{a}$ Electronic mail: jorge.garcia@uam.es

${ }^{b}$ Present address: Hewlett-Packard Research Laboratory, Palo Alto, CA.

${ }^{c)}$ Present address: Lehrstul für Werkstoffe der Electrotechnik University, Bochum, Germany.

${ }^{d)}$ Present address: Sektion Physik, LMU München, Geschwister-Scholl-Platz 1, 80539 München, Germany.
}

20 to $75 \AA$ ) at the same temperature, rotating the wafer to get uniform capping layer. The samples are cooled down under arsenic beam flux. AFM measurements are carried out at ambient conditions with a Nanoscope III AFM (tapping mode).

The PL measurements were done using an $\mathrm{Ar}^{+}$laser tuned at $514.4 \mathrm{~nm}$ focused on the sample to a spot size of $\approx 100 \mu \mathrm{m}$. The luminescence is dispersed by a $0.85 \mathrm{~m}$ double spectrometer, and detected with a liquid nitrogen cooled Ge detector with a spectral resolution of $0.5 \mathrm{~nm}$. The samples are cooled down to $15 \mathrm{~K}$ in a liquid helium cryostat.

Figure 1 shows three $1 \mu \mathrm{m}$ size AFM images of a $20 \AA$ GaAs capped sample for different positions on the wafer. A low InAs coverage region which gives a smooth capping with monolayer height $(2.8 \AA)$ fluctuations [Fig. 1(a)] because there are no QDs; a medium InAs coverage which lead to well resolved islands [Fig. 1(b)], and the higher InAs coverage areas where a rough surface $(\approx 40 \AA)$ is present [Fig. $1(\mathrm{c})$ ]. For InAs island densities $\leqslant 5 \cdot 10^{9} \mathrm{~cm}^{-2}$ it is possible to observe [Fig. 1(b)] well defined elongated partially capped islands (PCI). Sizes are listed in Table I.

Comparison of the island densities in an uncapped reference sample and capped ones grown under the same conditions shows similar densities. Therefore the InAs islands are the origin of these PCI. Cross section TEM taken under weak beam conditions, shows InAs rich regions in the center of the PCI in agreement with the AFM observations.

Once the PCI are close enough it is not possible to distinguish them because they overlap. The island density increases abruptly from $\approx 1.6 \mathrm{ML}$ InAs critical coverage $^{2}$ for a growth temperature of $530^{\circ} \mathrm{C}$. The maximum PCI density ever observed $\left(8 \cdot 10^{9} \mathrm{~cm}^{-2}\right)$ agrees with the maximum estimated value $\left(1 \cdot 10^{10} \mathrm{~cm}^{-2}\right)$ calculated by packing $130 \times 75$ $\mathrm{nm}^{2}$ PCI.

Flat surfaces between island correspond to areas in which the GaAs thickness is close to the nominally deposited one. This was confirmed by high resolution cross section TEM.

Figure 2(a) is an AFM image of a $65 \AA$ GaAs capped sample. The contour profiles shown in Fig. 2(b) are taken 

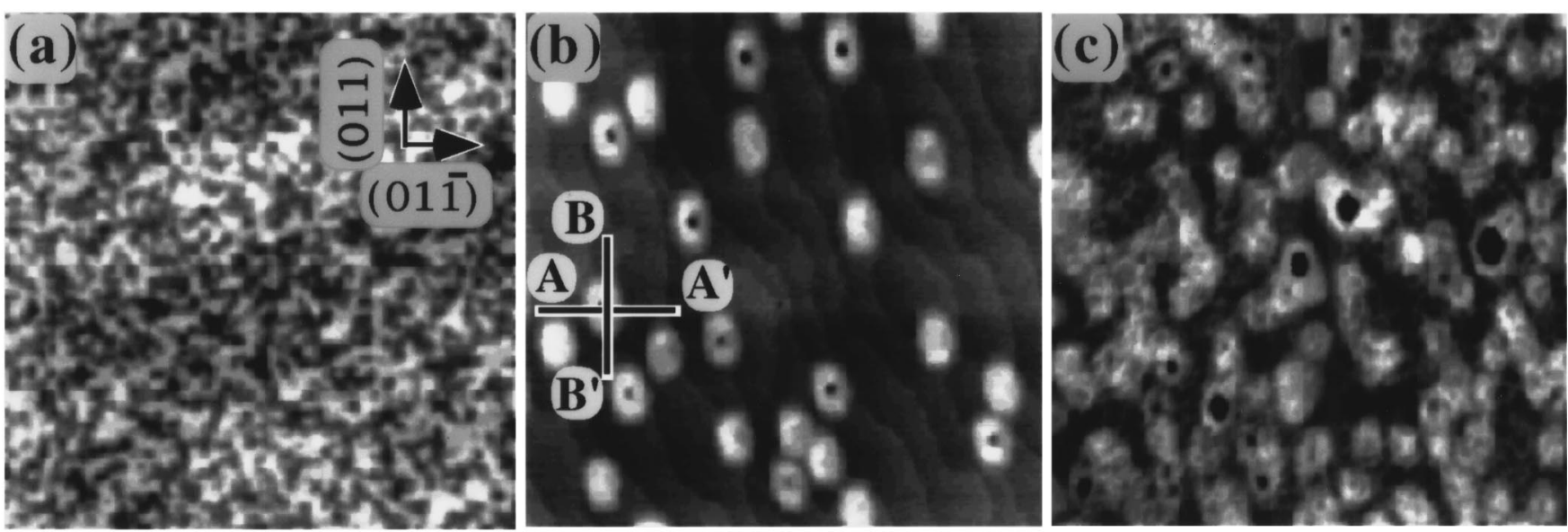

FIG. 1. $1 \mu \mathrm{m}$ size AFM images with different InAs island density along the [011] direction covered with $20 \AA$ GaAs. (a) 1 ML high fractal shaped islands on the surface of the low In coverage region. (b) Medium QD density. The holes are $\approx 13 \AA$ deep. (c) High density InAs island region.

parallel and perpendicular to $\langle 011\rangle$ directions for samples with 20 and $65 \AA$ capping layers. Although the uncapped original dot is lens shaped, the PCI are $\approx 1.7$ times larger in the [011] direction showing a clear anisotropy in the redistribution of the material. The thickness profiles are vertically shifted setting the flat regions to the nominal deposited thickness and plotted together with the uncovered dot profile (center).The profiles for the 20 and the $65 \AA$ capped samples show that there is a large amount of InAs redistribution. The volume of material around the original uncapped InAs dot island is approximately the same as the volume of the PCI formed. The PCI present a craterlike depression in the middle for the $20 \AA$ capping layer which become a clear hole for $40 \AA$ and change for higher $(50 \AA)$ GaAs thickness into a $(\approx 5 \AA)$ depression. This feature disappears for thicker capping layers.

The AFM profiles shown in Fig. 2 indicate that the InAs islands covered with a $20-\AA$-thick GaAs layer have reduced their size from $\approx 100$ to $\approx 20 \AA$. The material surrounding this type of PCI probably consists of $\operatorname{In}_{x} \mathrm{Ga}_{1-x}$ As. A $65 \AA$ GaAs capping layer completely covers the InAs island. How- ever, the height of the InAs island underneath the capping layer is still unknown.

It is legitimate to ask whether the snapshot picture given by the present growth interrupted experiments is still valid during a continuous overgrowth of a thick capping layer. TEM measurements ${ }^{17}$ show a height reduction $(\approx 50 \%)$ in GaAs capped InAs QDs indicating that shape and composition changes of the PCI are still present during the deposition of thick cap layers.

There is a small fraction $(<1 \%)$ of these PCI that present a bigger hole in the center which goes almost to the base of the dots [Fig. 2(a)]. The sides of the faceted hole in the center of these PCI are identical to that of the QD base observed by TEM.

Both the size changes and compositional changes are reflected in the PL spectra shown in Fig. 3. The spectra are taken at $15 \mathrm{~K}$ and with $23 \mathrm{~mW}$ in samples with the same PCI density. Spectrum 1 was obtained from a sample where the QDS have been etched away. The broad PL line at $1.15 \mathrm{eV}$ is associated with the substrate. A narrow peak [full width half maximum $(\mathrm{FWHM}) \approx 40 \mathrm{meV}]$ located at $0.971 \mathrm{eV}$ appears
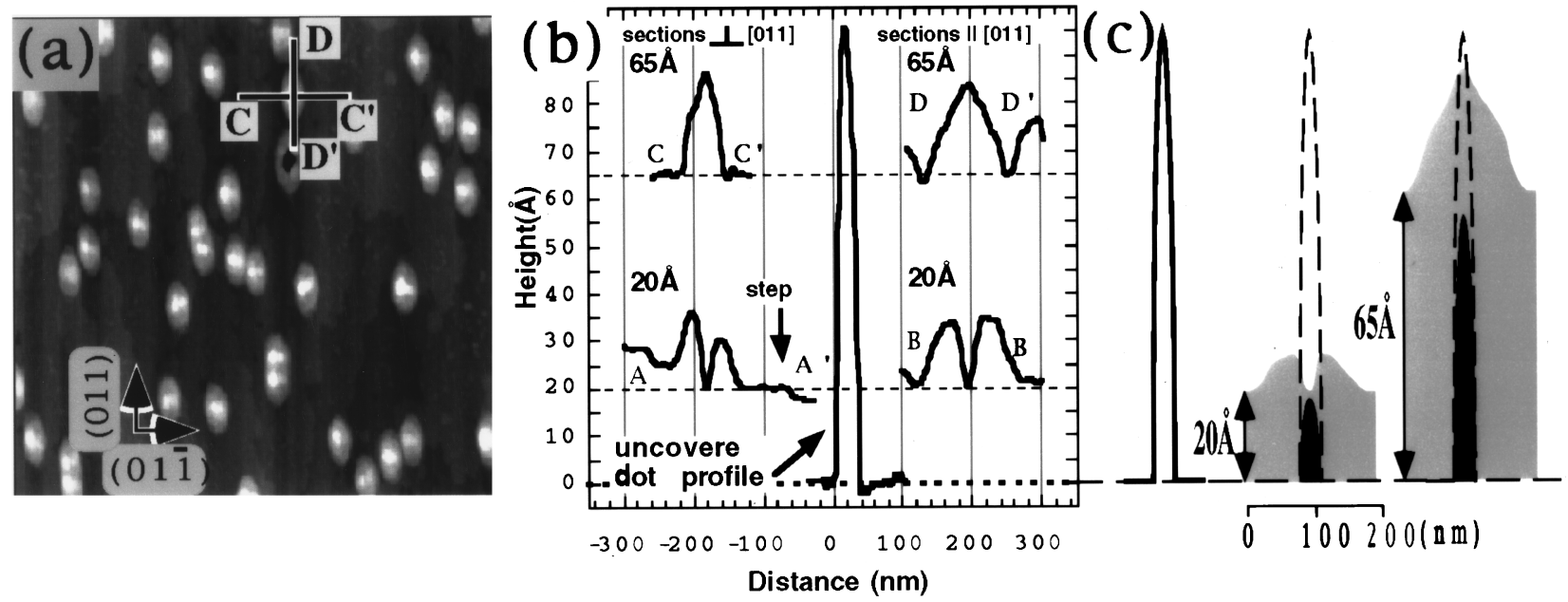

FIG. 2. (a) $1 \mu \mathrm{m}$ size AFM image of a sample covered with a $65 \AA$ GaAs capping layer. (b) The profiles are plotted together with those from Fig. 1 and with an uncovered QD. The plots have been vertically shifted according to the nominal GaAs deposited thickness. (c) Profiles are schematically shown using previously reported cross sectional transmission electron microscopy measurements (Ref. 17). 
TABLE I. Dimensions (nm) of the partially covered islands (PCI) for different nominal GaAs thickness. The height is measured above the film capping surface in between the PCI.

\begin{tabular}{lcccccc}
\hline \hline $\begin{array}{l}\text { GaAs } \\
\text { cap (nm) }\end{array}$ & 0 & 2 & 4 & 5 & 6.5 & 7.5 \\
\hline$[011]$ & $34 \pm 6$ & $110 \pm 8$ & $132 \pm 7$ & $137 \pm 10$ & $120 \pm 6$ & $130 \pm 10$ \\
{$[011]$} & $34 \pm 7$ & $70 \pm 9$ & $62 \pm 6$ & $86 \pm 5$ & $74 \pm 7$ & $85 \pm 5$ \\
Height & $10 \pm 1$ & $1.5 \pm 0.2$ & $1.5 \pm 0.2$ & $2.1 \pm 0.2$ & $2.1 \pm 0.2$ & $2.3 \pm 0.2$ \\
\hline \hline
\end{tabular}

(B) for the $50 \AA$ sample. This coverage coincides with the observed disappearance of the craterlike hole in the PCI center. For higher coverage $(65 \AA)$ this peak shifts to lower energies [(C) and (D)]. This could be due to both an increase in the QDs thickness and also to an increase of the In segregation in the region outside the QDs. These spectra show remarkably strong luminescence intensities for the surface QDs suggesting that carrier trapping by the QDs is more efficient than by surface states. The temperature dependence of the surface QDs indicates that thermal ionization of the carriers is not dominant even at room temperature.

The importance of In interdiffusion and surface diffusion is illustrated by studying the luminescence of two samples capped with a $65 \AA$ GaAs layer. One sample was cooled down immediately after the end of the growth (spectrum 3 ) and the other was annealed $25 \mathrm{~s}$ at $530{ }^{\circ} \mathrm{C}$ after the capping process was completed (spectrum 4). Both samples show a double peak structure with an energy splitting of $86 \mathrm{meV}$. These two lines correspond, respectively, to the ground state and first excited state of the QDs. ${ }^{3}$ The redshifted PL spectrum of the annealed sample together with the AFM mea-

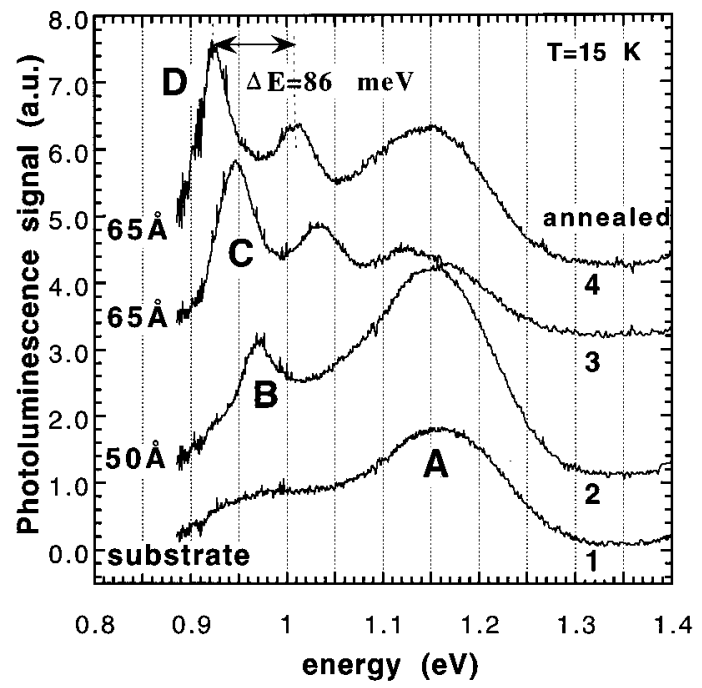

FIG. 3. $15 \mathrm{~K}$ photoluminescence spectra as a function of GaAs cap. The substrate signal is shown in spectrum 1. Spectra 3 and 4 show the effects of annealing. sured reduction of $30 \%$ in height of the PCI suggest as a primary reason for the observed redshift an enhanced In intermixing in the vicinities of the QDs. The marked narrowing $(\approx 30 \%)$ of the PL linewidth in the annealed sample spectrum also indicates an improved homogeneity in the QDs or capping layer composition.

Large changes in the dimensions, shapes, and composition of InAs islands deposited on GaAs are observed during the initial stages of the GaAs overgrowth which is used to change the islands into QDs. These changes are attributed to the regrowth kinetics that involve In diffusion and In and $\mathrm{Ga}$ surface exchange reactions. Such changes are intrinsic to the nonequilibrium nature of the MBE growth process and are probably present in other QD systems. The capping of the InAs islands is completed after $\approx 5 \mathrm{~nm}$ of GaAs deposition and allows the observation of the QDs luminescence states indicating that the QDs are remarkably insensitive to surface recombination effects.

The authors want to acknowledge M. Wendman for providing the special tips for AFM. This work was supported by QUEST, the National Science Foundation Science and Technology center (DMR No. 91-20007), the Alexander von Humobld foundation (KHS), the Brazilian agency $\mathrm{CNPq}$ (GMR), the Max Planck research award, the BMBF 01 BM $623(\mathrm{AL})$ and the Spanish Ministry of Education and Science (JMG).

${ }^{1}$ D. Leonard, M. Krishnamurthy, C. M. Reaves, S. P. Denbaars, and P. M. Petroff, Appl. Phys. Lett. 63, 3203 (1993).

2 D. Leonard, K. Pond, and P. M. Petroff, Phys. Rev. B 50, 11687 (1994); M. Grundmann, J. Christen.

${ }^{3}$ S. Raymond, S. Fafard, P. J. Poole, A. Wojs, P. Hawrylak, S. Charbonneau, D. Leonard, R. Leon, P. M. Petroff, and J. L. Merz, Phys. Rev. B 54, 11548 (1996); K. H. Schmidt, G. Medeiros-Ribeiro, M. Oestreich, P. M. Petroff, and G. H. Döhler, ibid. 54, 11346 (1996).

${ }^{4}$ K. H. Schmidt, G. Medeiros-Ribeiro, J. M. García, and P. M. Petroff, Appl. Phys. Lett. 70, 1727 (1997).

${ }^{5}$ M. E. Rubin, G. Medeiros-Ribeiro, J. J. O'Shea, M. A. Chin, E. Y. Lee, P. M. Petroff, and V. Narayanamurti, Phys. Rev. Lett. 77, 5268 (1996).

${ }^{6}$ G. Yusa and H. Sakaki, Appl. Phys. Lett. 70, 345 (1997).

${ }^{7}$ N. Kirstaedter, O. G. Schmidt, N. N. Ledentsov, D. Bimberg, V. M. Ustinov, A. Egorov Yu, A. E. Zhukov, M. V. Maximov, P. S. Kop'ev, and Zh. I. Alferov, Appl. Phys. Lett. 69, 1226 (1996).

${ }^{8}$ H. Shoji, Y. Nakata, K. Mukai, Y. Sugiyama, M. Sugawara, N. Yokoyama, and H. Ishikawa, Jpn. J. Appl. Phys., Part 2 35, L903 (1996).

${ }^{9}$ J.-Y. Marzin and G. Bastard, Solid State Commun. 92, 437 (1994).

${ }^{10}$ M. Grundmann, O. Stier, and D. Bimberg, Phys. Rev. B 52, 11969 (1995).

${ }^{11}$ J. M. Moison, F. Houzay, F. Barthe, L. Leprince, E. André, and O. Vatel, Appl. Phys. Lett. 64, 196 (1994).

${ }^{12}$ N. P. Kobayashi, T. R. Ramachandran, P. Chen, and A. Madhukar, Appl. Phys. Lett. 68, 3299 (1996)

${ }^{13}$ N. N. Ledentsov, V. A. Shchukin, M. Grundmann, N. Kirstaedter, and D. Bimberg, Phys. Rev. B 54, 8743 (1996).

${ }^{14}$ O. Brandt, L. Tapfer, K. Ploog, R. Bierwolf, and M. Hohenstein, Appl. Phys. Lett. 61, 2814 (1992).

${ }^{15}$ Q. Xie, P. Chen, and A. Madhukar, Appl. Phys. Lett. 65, 2051 (1994).

${ }^{16} \mathrm{H}-\mathrm{W}$. Ren, K. Nishi, S. Sugou, M. Sugisaki, and Yasuaki, Jpn. J. Appl. Phys., Part 1 36, 4118 (1997).

${ }^{17}$ D. Leonard, M. Krishnamurthy, S. Fafard, J. L. Merz, and P. M. Petroff, J. Vac. Sci. Technol. B 12, 1063 (1994). 